\title{
Estudio clínico controlado aleatorizado abierto de la eficacia y seguridad de "gentamicina-clindamicina" Vs. "pefloxacina- metronidazol" en el tratamiento de la endometritis post-cesárea
}

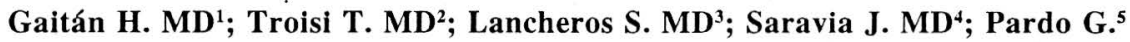

RESUMEN: Con el fin de comparar la eficacia y seguridad de dos regímenes terapéuticos (clindamicina-gentamicina y pefloxacinametronidazol) en el tratamiento de la endometritis post-cesárea, se incluyeron 71 pacientes que fueron sometidas a parto abdominal de urgencia en el Instituto Materno Infantil del $1^{\circ}$ de septiembre de 1993 al 31 de agosto de 1994 y que presentaron endometritis post-cesárea diagnosticada por la presencia de tres de nueve criterios utilizados en el Servicio de Sépticas. Se asignaron de manera aleatoria a uno de los dos grupos de tratamiento: gentamicina-clindamicina o metronidazol-pefloxacina. Se estudió la eficacia terapéutica mediante la comparación del tiempo de desaparición de la fiebre, la evolución clínica, los fracasos terapéuticos y las complicaciones. También se evaluó la seguridad de los dos regímenes. Se aplicó la prueba t de student y Chi cuadrado para significación estadística (a=0.05).

RESULTADOS: La seguridad se determinó en 68 pacientes que asistieron al control el día 5 de tratamiento y la eficacia en 63 pacientes que asistieron al control del día 7. Los cultivos de endometrio mostraron gérmenes anaerobios en el $\mathbf{4 8 \%}$ de las pacientes y se aislaron en promedio 1.9 gérmenes por paciente. No hubo diferencias entre los dos grupos de tratamiento en cuanto a desaparición de la fiebre ( $p=0.82$ ), tasa de mejoría $(\mathrm{p}=\mathbf{0 . 4 9})$, complicaciones $(\mathrm{p}=\mathbf{0 . 4 4})$, seguridad $(\mathrm{p}=\mathbf{0 . 9 4})$. La sensibilidad antibiótica de los gérmenes aerobios fue alta para los antibióticos utilizados y no se encontró b lactamasa en los cuatro casos en los que se aisló $\mathbf{N}$. gonorroheae.

CONCLUSIONES: La asociación clindamicina-gentamicina fue tan eficaz como la asociación pefloxacina-metronidazol en el tratamiento de la endometritis post-cesárea por lo que ésta última asociación se podría tener como una alternativa terapéutica.

PALABRAS CLAVES: Infección post-cesárea, endometritis, etiología, tratamiento, complicaciones.

\section{SUMMARY}

OBJECTIVE: Comparison between two therapeutics schedules Gentamicin plus and Pefloxacine plus metronidazol in the post-cesarean section endometritis.

DESIGN: One open randomized clinical trial was started. There were 71 women, who were undergone to urgency cesarean section in the Instituto Materno Infantil, from September 1st 1993 to August 31th 1994 who developed endometritys. The diagnostic was done with three of nine criteria's that we used in the septic ward in our Hospital. They were randomized in two groups of treatment, clindamicyn plus gentamicin or Pefloxacine plus Metronidazol. We studied the therapeutic efficacy between the regimens with the comparison of time of the fever effervescence, clinical curse, therapeutic falls and complications. The safety of the treatments were studied too. Statistical comparisons was done with $t$ student for continuos variables and chi square for discrete variables (a0.05).

RESULTS: The safety was studied in 68 patients who assisted to control on 5 th day of treatment and the efficacy in 63 patients who assisted to controls on 7 th day. The endometrial cultures showed anaerobic microorganisms in $48 \%$ of the patients and there was 1.9 microorganisms per patient. There was not differences between two therapeutic regimens about effervescence fever ( $p=0.94$ ), improvement rate $(p=0.49)$, complications $(p=0.44)$ and safety $(0.94)$. The antibiotic accuracy to aerobic germens was high for the two regimens studied, although we did not find $b$ lactamic resistance in the four cases of $\mathrm{N}$ gonorrhoeae.

CONCLUSIONS: Clindamycin-Gentamicin regimen was too successful like the association Pefloxacine-Metronidazol in the treatment of post-cesarean endometritis, showed the last regimen to be an alternative therapeutic, especially in cases of severe infection or fault on the first regimen.

KEY WORDS: post-cesarean infection, endometritis, microbiology, treatment, complications.

Profesor Asistente del Departamento de Ginecología y Obstetricia de la Universidad Nacional de Colombia. Coordinador del Servicio de Sépticas del Instituto Materno Infantil de Santa Fe de Bogotá.

Residente de segundo año de entrenamiento en el Departamento de Obstetricia y Ginecología de la Universidad Nacional de Colombia.
Residente de segundo año de entrenamiento en el Departamento de Obstetricia y Ginecología de la Universidad Nacional de Colombia.

$4 \quad$ Profesor Asociado de Medicina Interna. Unidad de Infectología. Departamento de Medicina. Facultad de Medicina, Universidad Nacional de Colombia.

5 Instructora Asociada. Departamento de Medicina Interna. Unidad de Infectología. Facultad de Medicina. Universidad Nacional de Colombia. 


\section{Introducción}

El advenimiento de mejores técnicas de evaluación fetal y perinatal (1-3) conlleva a un aumento progresivo de la realización de cesáreas. El parto abdominal no sólo aumenta el riesgo de infección sino también la severidad de la misma, lo que explica el aumento de la infección puerperal como causa de morbi-mortalidad materna en los últimos años en el Instituto Materno Infantil (4).

En 1993, en el Instituto Materno Infantil, aproximadamente el $7 \%$ de las mujeres sometidas a cesárea presentaban infección, siendo la localización más frecuente el endometrio, seguido por la herida quirúrgica y el parametrio (4). Actualmente de 300 ingresos registrados en el Servicio de Sépticas el $40 \%$ corresponde a infección post-cesárea. (Informe interno servicio estadístico IMI).

La gravedad de la infección post-cesárea ha sido relacionada con la presencia de gérmenes anaerobios, normalmente existentes en el tracto genital inferior, los cuales invaden la herida quirúrgica en donde se encuentran tejidos desvitalizados o isquémicos, con hematomas y cuerpos extraños (suturas) que facilitan su desarrollo. Junto con los microorganismos anaerobios se encuentran aerobios y se produce una infección polimicrobiana mixta con cuadros más graves y difíciles de manejar (7-9).

El tratamiento de la endometritis post-cesárea se basa en el diagnóstico temprano de la infección y el suministro de antibióticos que cubran tantos gérmenes anaerobios como aerobios implicados en esta severa complicación.

Con respecto al tratamiento antibiótico de la endometritis post-cesárea, la asociación de gentamicinaclindamicina es la antibiótico-terapia más utilizada y ha sido el patrón de comparación desde 1977 cuando demostró disminuir la tasa de fracasos y complicaciones frente a las asociaciones utilizadas en esa época (10-13).

Con la aparición de nuevos antibióticos como la pefloxacina, una quinolona fluorada que actúa bloqueando la replicación del DNA por inhibición de la DNA girasa bacteriana especialmente de Pseudomonas, Estafilococos, Enterobacterias y Neisseria sp entre otros, se tiene otra alternativa de tratamiento diferente a los aminoglucósidos con menores efectos nefro y ototóxicos. Por otra parte, el metronidazol, un imidazol activo en infecciones causadas por anaerobios de los géneros Bacteroides y Clostridium frecuentemente implicados en la infección post-cesárea, registra un menor riesgo de producir efectos secundarios importantes como la enterocolitis pseudomembranosa asociada al uso de la clindamicina y aparece como un buen complemento de las quinolonas.

El objetivo de este estudio fue evaluar la eficacia y seguridad de la asociación gentamicina-clindamicina $(\mathrm{G}+\mathrm{C})$ comparada con la asociación de pefloxacinametronidazol $(\mathrm{P}+\mathrm{M})$ en el tratamiento de las infecciones polimicrobianas causantes de la endometritis post-cesárea en busca de una nueva alternativa terapéutica para estas pacientes, principalmente aquellas con cuadros más graves y severos.

\section{Materiales y métodos}

El presente estudio se realizó en el Servicio de Sépticas del Instituto Materno Infantil entre septiembre de 1993 y agosto de 1994. El Instituto Materno Infantil es un centro de referencia para embarazos de alto riesgo donde se atienden pacientes de escasos recursos, las cuales presentan un alto riesgo de infección (5).

Toda paciente hospitalizada con sospecha de endometritis post-cesárea fue remitida al Servicio de Sépticas donde se realizó una historia clínica y examen físico completo para confirmar la presencia de infección.

Las pacientes, previo consentimiento informado, fueron incluidas en el estudio cuando cumplían los siguientes tres criterios: 1. Dieciséis años o más. 2. Cesárea de Urgencia en el Instituto Materno Infantil. 3. Infección endouterina posterior (endometritis).

Las pacientes fueron excluidas cuando presentaban alguno de los siguientes criterios: 1. Conocida hipersensibilidad o historia de intolerancia a los macrólidos, aminoglucósidos, quinolonas o imidazoles. 2. Evidencia de infección antes de la cesárea (corioamnionitis, infección urinaria, etc). 3. Ruptura prematura de membranas (mayor 24 horas de evolución). 4. Antibioticoterapia en las dos semanas anteriores a la inclusión. 5. Enfermedad hepática o renal (transaminasas mayores a $200 \mathrm{U}$ o creatinina mayor a $1.5 \mathrm{mg} \%$ ). 6. Cáncer o alguna enfermedad autoinmune o tratamiento inmunosupresor. 7. Falta de sensibilidad a los Antibióticos en estudio de los gérmenes aislados en el cultivo de endometrio. 8. Requerir antibióticos diferentes a los propuestos para el estudio.

El protocolo del estudio fue aprobado por el Comité Etico del Instituto Materno Infantil y el de la Universidad Nacional de Colombia.

Las pacientes se asignaron aleatoriamente en uno de los grupos de estudio cuando cumplieron los criterios de inclusión. La aleatorización se realizó en bloques por medio de una tabla.

El primer grupo recibió gentamicina $(\mathrm{G})$ a razón de 4 mg por kilogramo de peso al día cada 12 horas y clindamicina (C) a razón de $600 \mathrm{mg}$ cada 6 horas. Las drogas se administraron por vía intravenosa hasta que la paciente completó 24 horas afebril, posteriormente se cambiaron a gentamicina $120 \mathrm{mg}$ IM cada 12 horas y clindamicina $600 \mathrm{mg}$ VO cada 8 horas hasta completar 7 días de tratamiento. Al segundo grupo se le administró metronidazol (M) $500 \mathrm{mg}$ cada 8 horas y pefloxacina (P) $400 \mathrm{mg}$ cada 12 horas. Los fármacos se administraron por vía intravenosa inicialmente, al completar la paciente 24 horas afebril se cambió a metronidazol $500 \mathrm{mg}$ cada 8 horas y pefloxacina $400 \mathrm{mg}$ cada 12 horas por vía oral, para un total de 7 días. No fue necesario ajustar la dosificación de los fármacos puesto que no se recibieron pacientes con falla renal o insuficiencia hepática.

Una vez incluida la paciente se tomaron al menos dos muestras para cultivo aerobio y anaerobio de endometrio y de sangre (2). Se elaboró examen hematológico (cuadro hemático completo con conteo celular diferencial y velocidad de sedimentación globular), química sanguínea (transaminasa glutámico oxalacética y pirúvica, bilirrubinas, fosfatasa alcalina, nitrógeno uréico, creatinina, 
glicemia, proteínas totales, tiempo de protrombina y tiempo parcial de tromboplastina) y uroanálisis. El cuadro hemático se repitió al quinto día del estudio.

El diagnóstico de endometritis post-cesárea, definida como la infección localizada en el endometrio causada por gérmenes del tracto genital femenino en una paciente sometida a operación cesárea, se basó en la presencia de tres de los siguientes criterios utilizados corrientemente en el Servicio de Sépticas del Instituto Materno Infantil para Infección puerperal: Fiebre (temperatura oral > $38.0^{\circ} \mathrm{C}$ ), taquicardia ( $>90$ pulsaciones por minuto), loquios fétidos o purulentos, dolor uterino, cuello uterino abierto $(>2 \mathrm{~cm})$, masas uterinas dolorosas asociadas a loquios fétidos, leucocitosis $>15.000$, neutrofilia o presencia de cayados y velocidad de sedimentación globular $>40 \mathrm{~mm} /$ hora.

Los cultivos para diagnóstico microbiológico antes de iniciar la terapia antibiótica. Si al tercer día persistían los signos de infección se repitió el cultivo de endometrio. En las pacientes que presentaron bacteriuria inicial, a pesar de tomar la muestra con sonda, se hizo urocultivo y antibiograma.

Las muestras de sangre iniciales se obtuvieron antes de la iniciación de la terapia. Los hemocultivos para gérmenes aerobios fueron tomados en una proporción 10$20 \%$ muestra y medio de cultivo. Se utilizaron tripticasa soya base (BBL), glucosa (BBL), suplementado con vitaminas y aminoácidos (Vitos ${ }^{\mathrm{r}}$, Oxoid), hemina y vitamina $\mathrm{K}$ con incubación a $35^{\circ} \mathrm{C} \pm 2$ por 10 días con repiques y Gram a las 24 horas.

Las muestras de secreción endometrial se obtuvieron mediante una modificación de la técnica de Arredondo y colaboradores (15): previo lavado de exocervix con solución salina, se pasa un tubo orotraqueal No. 14 por el endocervix hasta cavidad endometrial, a través de él se pasa una sonda nasogástrica para adultos No. 14 y por ésta una sonda nasogástrica para niños No. 6, para aspiración del contenido endometrial por medio de una jeringa o haciendo lavado de la cavidad con solución salina estéril cuando es difícil obtener material. La sonda sellada distalmente, junto con la jeringa se transportaron inmediatamente al laboratorio de Infectología del Departamento de Medicina Interna del Hospital San Juan de Dios.

Los cultivos se realizaron en los medios apropiados para detectar gérmenes aerobios, microaerófilos y anaerobios (caldo tripticasa soya (BBL), trioglicolato 135 $\mathrm{C} \sin$ indicador (BBL) y medio de carne cocida (Oxoid) prerreducidos, agar sangre (sangre de cordero al 5\%), agar brucella (BBL), agar Mac Conkey (Oxoid), agar Thayer Martin (BBL), agar Wilkin s Chalgren (Oxoid) suplementado con hemina y vitamina $\mathrm{K}$ y/o N-G (Oxoid) y su identificación final se hizo con Anident (Oxoid). Todos los procedimientos se hicieron de acuerdo con las recomendaciones del productor y los gérmenes aislados fueron identificados por los métodos convencionales.

La susceptibilidad antimicrobiana para la pefloxacina y la gentamicina se llevó a cabo para todos los patógenos aerobios obtenidos en endometrio o sangre. Las pruebas de susceptibilidad se elaboraron por el método Kirby Bauer.
La evaluación clínica se repitió el día 3, 5 y 7 de la terapia y la respuesta se clasificó como "curación" cuando todos los signos y síntomas asociados a la infección desaparecieron o disminuyeron y no hubo ningún síntoma nuevo en el seguimiento post-tratamiento), "Mejoría" cuando hubo persistencia de uno de los criterios diagnósticos de infección y "Falla" cuando se observaron persistencia o empeoramiento de los signos o síntomas clínicos de infección, o aparición de nuevos signos o síntomas durante la hospitalización. La mayoría de las pacientes estuvieron hospitalizadas 7 días para asegurar el cumplimiento del protocolo.

La seguridad del tratamiento se evaluó como "Excelente" cuando no se presentaron efectos adversos, "Bue$n a$ " los efectos adversos fueron leves y no obligaron a suspender el tratamiento, "Regular" cuando los efectos adversos obligaron a instaurar medidas terapéuticas para continuar el tratamiento y "Mala" cuando el efecto adverso fue tan severo o serio que obligó a suspender el tratamiento.

El análisis estadístico se realizó generando una base de datos en Dbase IV, posteriormente se tradujo al formato SPSSPC 6.0 para Windows. Se elaboró una comparación por el método de chi cuadrado para variables categóricas y el test $\mathrm{t}$ de student para variables continuas. Una $\mathrm{p}<0.05$ fue tomada como significativa. Los resultados se analizaron con el propósito de comparar si el tratamiento estudiado fue tan bueno como el utilizado convencionalmente.

\section{Resultados}

Durante un período de 11 meses, 71 pacientes ingresaron al estudio. De éstas, 36 pacientes recibieron $\mathrm{C}+\mathrm{G}$ y $35 \mathrm{P}+\mathrm{M}$.

Debido a la ausencia de loquios fétidos se excluyeron 3 pacientes, todas ellas pertenecientes al grupo $\mathrm{P}+\mathrm{M}$. Del total de pacientes, 5 no asistieron al control del séptimo día, 3 del grupo $\mathrm{G}+\mathrm{C}$ y 2 del grupo $\mathrm{P}+\mathrm{M}$.

Se encontró diferencia significativa entre los grupos tratados con respecto al promedio de edad $(\mathrm{p}=0.04)$ y no hubo diferencias significativas con respecto a las otras variables estudiadas. (Tabla 1).

Del total de pacientes el $57 \%$ tenían su primer embarazo y había signos de desnutrición leve a moderada en el $46 \%$ de ellas.

La indicación de la cesárea más frecuente fue la desproporción céfalo pélvica $(25 \%)$ seguida por la presentación de pelvis ( $21 \%$ ) y se utilizó anestesia regional en el $97 \%$ de las pacientes. (Tabla 2).

En cuanto a la evolución de la infección post-cesárea la fiebre apareció en las primeras 48 horas del post operatorio en el $80 \%$ de las pacientes $\left(X=38.67^{\circ} \mathrm{C}\right)$. (Tabla 3).

En el examen de sangre se detectó aumento de la velocidad de sedimentación globular $(X=51 \mathrm{~mm} / \mathrm{hora})$ y del recuento de neutrófilos ( $\mathrm{X}=80 \%$ ). (Tabla 4).

En la secreción endometrial se aislaron gérmenes en un $92 \%(65 / 71)$ de las muestras obtenidas, $48 \%$ anaerobios, para un promedio de 1.9 gérmenes por paciente. (Tablas 5-6-7). No se encontró b lactamasa en los 
Tabla 1

COMPARACION DE LAS CARACTERISTICAS GENERALES DE LAS PACIENTES

\begin{tabular}{|c|c|c|c|c|c|}
\hline Variable & $\begin{array}{c}\text { Promedio } \\
\text { Grupo } \\
\text { C+G }\end{array}$ & $\begin{array}{c}\text { No/Total } \\
\text { Grupo C+G }\end{array}$ & $\begin{array}{c}\text { Promedio } \\
\text { Grupo } \\
\text { P+M }\end{array}$ & $\begin{array}{l}\text { No. Total } \\
\text { Grupo PM }\end{array}$ & $\mathbf{P}$ \\
\hline $\begin{array}{l}\text { Edad (años) } \\
\text { Primigestantes } \\
\text { Nivel socioeconómico } \\
\quad \text { Medio } \\
\quad \text { Bajo } \\
\text { No. Controles prenatales } \\
\text { Edad Gestacional (Semanas) } \\
\text { Factores de Riesgo } \\
\quad \text { Edad (extremos) } \\
\quad \text { Hipertensión } \\
\quad \text { DNT leve } \\
\quad \text { DNT moderada } \\
\quad \text { Flujo Patológico } \\
\text { Ruptura Prematura Membranas } \\
\text { (Horas) } \\
\text { No. de tactos vaginales } \\
\text { Dilatación Cervical precesárea } \\
\text { (cm) }\end{array}$ & $\begin{array}{c}24.27 \\
- \\
- \\
- \\
4.2 \\
38.45 \\
- \\
- \\
- \\
- \\
- \\
2.97 \\
3.36 \\
5.13\end{array}$ & $\begin{aligned} & - \\
18 / 36 & (50 \%) \\
& \\
22 / 35 & (63 \%) \\
13 / 35 & (37 \%) \\
- & \\
- & \\
11 / 36 & (30 \%) \\
6 / 35 & (17 \%) \\
7 / 27 & (26 \%) \\
7 / 27 & (26 \%) \\
7 / 35 & (20 \%) \\
- & \\
- & \\
- & \end{aligned}$ & $\begin{array}{c}20.12 \\
- \\
- \\
- \\
3.7 \\
39.15 \\
- \\
- \\
- \\
- \\
- \\
1.93 \\
3.71 \\
5.75\end{array}$ & $\begin{aligned} 21 / 32 & - \\
& (66 \%) \\
23 / 31 & (74 \%) \\
8 / 31 & (26 \%) \\
- & \\
- & \\
18 / 32 & (56 \%) \\
7 / 32 & (22 \%) \\
6 / 23 & (26 \%) \\
3 / 23 & (13 \%) \\
10 / 32 & (31 \%) \\
- & \\
- & \\
- & \end{aligned}$ & $\begin{array}{l}0.32 \\
0.5 \\
0.2 \\
\\
0.03 \\
0.62 \\
0.98 \\
0.5 \\
0.29 \\
0.41 \\
0.58 \\
0.45\end{array}$ \\
\hline
\end{tabular}

Tabla 2

CARACTERISTICAS DE LA CESAREA

\begin{tabular}{|l|c|l|}
\hline Variable & Grupo A & Grupo B \\
\hline Tipo de anestesia & & \\
Regional & $36 / 36(100 \%)$ & $30 / 32(94 \%)$ \\
General & $0 / 36(0 \%)$ & $2 / 32(6 \%)$ \\
\hline Indicación de cesárea & & \\
\hline Sufrimiento fetal agudo & $5 / 36(14 \%)$ & $4 / 32(13 \%)$ \\
Embarazo gemelar & $6 / 36(17 \%)$ & $1 / 32(3 \%)$ \\
Desproporción cefalopélvica & $9 / 36(25 \%)$ & $7 / 32(22 \%)$ \\
Presentación anómala & $7 / 36(19 \%)$ & $7 / 32(22 \%)$ \\
Obito fetal & $0 / 36(0 \%)$ & $1 / 32(3 \%)$ \\
Iterativa & $3 / 36(8 \%)$ & $5 / 32(16 \%)$ \\
Alteración del Trabajo de Parto & $3 / 36(8 \%)$ & $4 / 32(13 \%)$ \\
Insuficiencia Placentaria & $2 / 36(6 \%)$ & $2 / 32(6 \%)$ \\
Macrosomía & $0 / 36(0 \%)$ & $1 / 32(3 \%)$ \\
Hipertiroidismo & $1 / 36(3 \%)$ & $0 / 32(0 \%)$ \\
\hline Complicaciones cesárea & $4 / 36(11 \%)$ & $1 / 32(3 \%)$ \\
\hline Estado del producto & & \\
Vivo, sano & $29 / 35(83 \%)$ & $25 / 32(78 \%)$ \\
Vivo, enfermo & $4 / 35(11 \%)$ & $7 / 32(22 \%)$ \\
Fallecido & $2 / 35(6 \%)$ & $0 / 32(0 \%)$ \\
\hline
\end{tabular}

cuatro casos en los que se aisló $N$. gonorroheae. La serología fue negativa en todas las pacientes. El urocultivo $y$ el hemocultivo fueron positivos en el $16 \%$ de las pacientes.

Inicialmente se diagnosticaron las siguientes complicaciones infecciosas de la cesárea: infección de la herida quirúrgica $(40 \% ; 27 / 68)$ y masa parametrial $(12 \% ; 8 / 68)$, las cuales fueron tratadas exitosamente. No hubo diferencias significativas entre los dos grupos $(\mathrm{p}=0.88$ y 0.56 respectivamente).

\section{Evolución clínica:}

- Duración de la fiebre: Las pacientes del grupo $\mathrm{C}+\mathrm{G}$ presentaron en promedio 3.48 días de fiebre, mientras que las del grupo $\mathrm{P}+\mathrm{M}$ obtuvieron promedios de 2.48 días, con una diferencia estadísticamente significativa $(\mathrm{p}=0.824)$.

Las cinco pacientes que fueron dadas de alta al quinto día fueron evaluadas clínicamente como "Curadas", ellas solicitaron su salida al investigador este día y no regresaron al control del séptimo día.

El séptimo día de tratamiento las pacientes fueron evaluadas clínicamente de la siguiente manera:

- Grupo terapéutico C+G: "Curación" en el 79\% (26/33), "Mejoría" en el 12\% (4/33) y "Falla" en el 9\% (3/33).

- Grupo terapéutico P+M: "Curación" en el 70\% (21/ $30)$, "Mejoría" en el 23\% (7/30) y "Falla" en el 7\% (2/ 30). (Tabla No. 8).

No hubo diferencia significativa entre los dos grupos $(\mathrm{p}=0.50)$. 
Tabla 3

CARACTERISTICAS CLINICAS

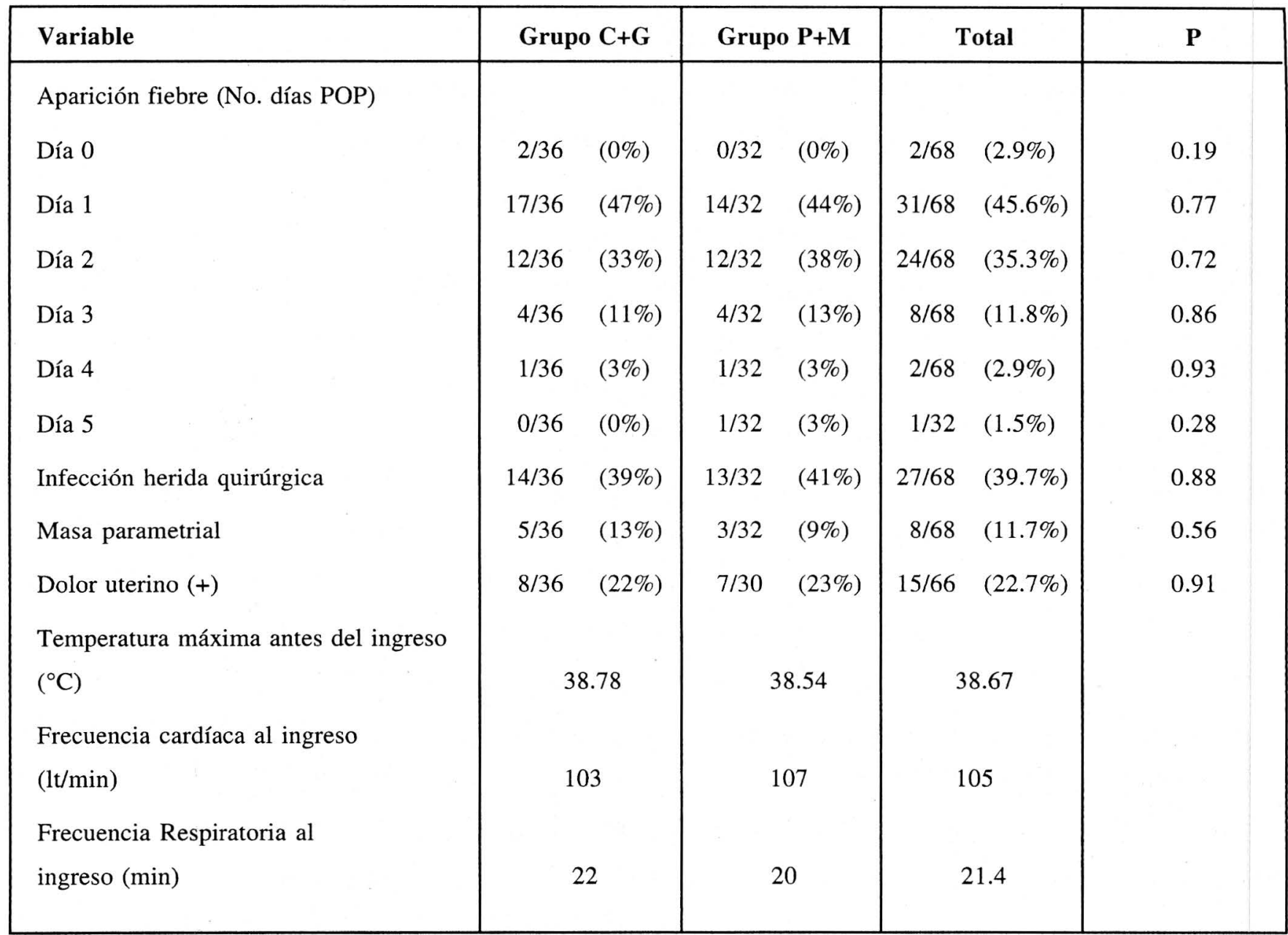

Tabla 4

HALLAZGOS DE LABORATORIO

\begin{tabular}{|l|l|l|l|}
\hline Variable & Grupo C+G & Grupo P+M & Grupo Total \\
\hline & & & \\
Hemoglobina gr \% & 10.7 & 11.5 & 11.1 \\
Leucocitos por mm ${ }^{2}$ & 13.663 & 13.053 & 13.376 \\
Neutrófilos \% & 80.08 & 80.90 & 80.47 \\
Linfocitos \% & 17.13 & 16.43 & 16.81 \\
Cayados \% & 1.11 & 1.09 & 1.10 \\
Plaquetas por mm ${ }^{2}$ & 25.053 & 26.346 & 24.643 \\
VSG mm/hora & 51.33 & 50.56 & 50.97 \\
Parcial de orina sugestivo de Infección & $26 / 36(72 \%)$ & $17 / 31(55 \%)$ & $43 / 67(64.1 \%)$ \\
Urocultivo (+) & $3 / 26(12 \%)$ & $4 / 17(23 \%)$ & $7 / 43(16.3 \%)$ \\
\hline
\end{tabular}


Tabla 5

GERMENES AISLADOS POR MUESTRA EN LA SECRECION ENDOMETRIAL

\begin{tabular}{|l|l|l|l|l|l|}
\hline Germen & No. & \% & Germen & No. & Anaerobios \\
\hline \multicolumn{1}{|c|}{ Aerobios } & & & & & \\
\hline S. aureus & 28 & 42 & Peptostreptococcus & 23 & 35 \\
S. epidermidis & 9 & 13 & Peptococcus & 5 & 8 \\
Streptococcus sp & 7 & 11 & C. perfringes & 3 & 5 \\
Streptococcus grupo A & 1 & 1 & B. fragilis & 18 & 28 \\
Streptococcus a hemolítico & 2 & 3 & P. melaninogenicus & 15 & 23 \\
Streptococcus fecalis & 2 & 3 & Fusobacterium & 1 & 1 \\
E. coli & 8 & 12 & Total & 65 & 100 \\
P. mirabilis & 2 & 3 & Candida albicans & 1 & 1 \\
H. alvei & 1 & 1 & Aerobios & & 51 \\
C. freundi & 1 & 1 & Anaerobios & & 1 \\
G. vaginalis & 2 & 3 & Hongos & & \\
N gonorrhoeae & 4 & 6 & & & \\
Corynebacterium genitarium & 1 & 1 & & & \\
\end{tabular}

Tabla 6

GERMENES AISLADOS POR MUESTRA DE SECRECION ENDOMETRIAL

\begin{tabular}{|c|c|c|}
\hline No. de Gérmenes & No. de Muestras & Porcentaje \\
\hline 1 & 20 & 29 \\
2 & 25 & 35 \\
3 & 13 & 19 \\
4 & 6 & 8 \\
5 & 1 & 1 \\
0 & 6 & 8 \\
\hline Total & 71 & 100 \\
\hline
\end{tabular}

Durante el tratamiento se presentaron 7 complicaciones: 4 casos de parametritis (12\%) en el grupo $\mathrm{C}+\mathrm{G}$ y 2 $(6 \%)$ casos en el grupo $\mathrm{P}+\mathrm{M}(\mathrm{p}=0.46)$. Además hubo un caso de absceso pélvico en el grupo $\mathrm{C}+\mathrm{G}$ que requirió laparotomía para drenaje y cambio de la terapia antibiótica para su mejoría.

Las pacientes excluidas por la ausencia de loquios fétidos evolucionaron hacia la "Mejoría" (2 casos) y hacia la "Curación" (1 caso) el séptimo día.

\section{Seguridad de la terapia}

La seguridad de la terapia se clasificó como "Excelente" en el $94 \%$ de las pacientes del grupo C+G y "Mala" en el $6 \%$. En este grupo, una paciente $(3 \%)$ presentó

Tabla 7

SENSIBILIDAD DE LOS GERMENES COMUNES ASILADOS EN LA SECRECION ENDOMETRIAL

\begin{tabular}{|l|c|c|c|c|}
\hline Germen & Clindamicina & Gentamicina & Pefloxacina & Penicilina \\
\hline S. Aureus & $25 / 28$ & $26 / 28$ & $27 / 28$ & $-*$ \\
S. Epidermidis & $9 / 9$ & $9 / 9$ & $8 / 9$ & $-*$ \\
Streptococcus & $7 / 9$ & $6 / 8$ & $-*$ & $-*$ \\
E. Coli & $-*$ & $6 / 8$ & $7 / 8$ & $-*$ \\
P. Mirabilis & $-*$ & $2 / 2$ & $2 / 2$ & $-*$ \\
N. Gonorroheae & $2 / 2$ & $2 / 2$ & $2 / 2$ & $4 / 4$ \\
\hline
\end{tabular}

* No se estudió sensibilidad a este antibiótico

^ Sólo se realizó sensibilidad a los antibióticos en estudio en 2 pacientes. 
Tabla 8

EVOLUCION DE LAS PACIENTES EVALUABLES EL SEPTIMO DIA DE TRATAMIENTO

\begin{tabular}{|c|c|c|c|c|c|}
\hline \multirow{2}{*}{\begin{tabular}{|r|} 
Variable \\
Evolución Clínica
\end{tabular}} & \multicolumn{2}{|c|}{$\begin{array}{c}\text { Grupo } \mathrm{C}+\mathrm{G} \\
\text { No./Total }\end{array}$} & \multicolumn{2}{|c|}{$\begin{array}{c}\text { Grupo P+M } \\
\text { No./Total }\end{array}$} & \multirow{2}{*}{$\begin{array}{l}\mathbf{P} \\
0.494\end{array}$} \\
\hline & $26 / 33$ & $(79 \%)$ & $1 / 30$ & $(70 \%)$ & \\
\hline Curación & $4 / 33$ & $(12 \%)$ & $7 / 30$ & $(23 \%)$ & \\
\hline Mejoría & $3 / 33$ & $(9 \%)$ & $2 / 30$ & $(7 \%)$ & \\
\hline Falla & & & & & \\
\hline \multicolumn{6}{|l|}{ Evolución clínica (revaluación) } \\
\hline Buena & $27 / 36$ & $(75 \%)$ & $25 / 32$ & $(78 \%)$ & 0.50 \\
\hline Regular & $1 / 36$ & $(3 \%)$ & $3 / 32$ & $(9 \%)$ & \\
\hline Fracaso & $5 / 36$ & $(14 \%)$ & $2 / 32$ & $(6 \%)$ & \\
\hline No evaluable (NHD) & $3 / 36$ & $(8 \%)$ & $2 / 32$ & $(6 \%)$ & \\
\hline \multicolumn{6}{|l|}{ Reacciones adversas } \\
\hline No & $32 / 34$ & $(94 \%)$ & $28 / 30$ & $(93 \%)$ & 0.57 \\
\hline Leve & $1 / 34$ & $(3 \%)$ & $2 / 30$ & $(7 \%)$ & \\
\hline Moderado & $1 / 34$ & $(3 \%)$ & $0 / 30$ & $(0 \%)$ & \\
\hline \multicolumn{6}{|l|}{ Impresión Clínica Seguridad } \\
\hline Excelente & $30 / 31$ & $(97 \%)$ & $27 / 28$ & $(96 \%)$ & 0.941 \\
\hline Buena & $1 / 31$ & $(3 \%)$ & $1 / 28$ & $(4 \%)$ & \\
\hline Mala & $0 / 31$ & $(0 \%)$ & $0 / 28$ & $(0 \%)$ & \\
\hline
\end{tabular}

diarrea durante la terapia que obligó a suspender los antibióticos, y otra paciente (3\%) presentó fiebre que se consideró como fiebre medicamentosa ya que desapareció al suspender el tratamiento.

En el grupo P+F la seguridad fue "Excelente" en el 93\% de los casos y "Buena" en el 7\%. En este grupo una paciente $(3 \%)$ desarrolló rash cutáneo y otra paciente (3\%) presentó náusea, que no obligaron a la suspensión del tratamiento.

\section{Discusión}

Basándonos en el estudio de endometritis post-cesárea realizado en el Instituto Materno Infantil en el año 1991, donde se obtuvo una prevalencia de endometritis en un $7 \%$ (1), y aceptándose una prevalencia de hasta un máximo del $13 \%$ y un a del 0.05 , una muestra de 68 pacientes se puede considerar como representativa del grupo de pacientes sometidas a cesárea en el IMI con infección endouterina. Es el número de pacientes incluidas en este estudio.

La distribución de la mayoría de las variables demográficas: factores de riesgo, indicación de la cesárea y sus complicaciones infecciosas, tipo de anestesia y estado del recién nacido fue simétrica en los dos grupos terapéuticos. En cuanto a la edad, las pacientes menores de 20 años quedaron aleatoriamente asignadas al grupo $\mathrm{P}+\mathrm{M}$, lo que pudo afectar los resultados finales ya que se ha reportado mayor tendencia de las pacientes adolescentes a desarrollar infecciones graves.

En cuanto al cuadro clínico hay aparición temprana de la fiebre ( $80 \%$ en los dos primeros días), curso diferente al descrito por autores norteamericanos, pero ya encontrado en el IMI anteriormente (16), lo que nos permite sugerir que las pacientes con temperatura mayor de $38.5^{\circ} \mathrm{C}$ en el primer día post-operatorio de la cesárea realizada en el Instituto Materno Infantil deben considerarse como posiblemente infectadas (9).

Aunque para otros autores el dolor uterino es considerado un signo cardinal en la endometriosis post-cesárea, en nuestras pacientes se evidenció baja frecuencia del dolor uterino (23\%), el cual, además es de difícil interpretación por el dolor en la herida quirúrgica asociado.

En este estudio los datos de laboratorio: neutrofilia y aumento de la velocidad de sedimentación fueron los mejores indicadores de infección en las pacientes con infección endouterina post-cesárea.

La asignación de las pacientes con endometritis postcesárea a los grupos terapéuticos se realizó de manera aleatoria excepto ẹn las primeras 6 pacientes que salieron del estudio por violación del protocolo, las cuales evolucionaron de manera satisfactoria con el tratamiento instaurado y fueron reemplazadas en su asignación por otras 6 pacientes. Por esta razón decidimos presentar los dos grupos con las p asociadas.

La comparación de los dos esquemas terapéuticos no mostró diferencias estadísticamente significativas en cuanto a eficacia ( $p>0.05)$.

Las características de estudio abierto y los diferentes observadores (residentes rotatorios y adjuntos o especialistas del servicio) pueden favorecer de alguna forma uno de los dos grupos de tratamiento y la subjetividad de sus observaciones -especialmente en algunas variables blandas como las características de los loquios y el dolor uterino, sesgo de medición-. Para asegurar una buena evaluación clínica se generó una segunda variable de desenlace, una - Revaluación - realizada por un médi- 
co clínico quien evaluó la información consignada en cada formulario, para decidir en cada caso si el resultado final de efectividad terapéutico era "Bueno", "Regular" o "Malo". En la revaluación se obtuvieron resultados idénticos a los anteriores en cuanto a las tasas de Curación y Mejoría y no se encontraron diferencias clínica o estadísticamente significativas.

Los otros parámetros de evaluación de eficacia como la duración de la fiebre fueron muy similares.

Las tasas de éxito, Curación más Mejoría, para los dos grupos terapéuticos fue del $91 \%$ para $\mathrm{C}+\mathrm{G}$ y de $93 \%$ para $\mathrm{P}+\mathrm{M}$ similares a las tasas de curación reportadas en otros estudios: $91 \%$ para sulbactam-ampicilina y gentamicinametronidazol (17), gentamicina-clindamicina (86\%), cefamandol $(82 \%)$ y moxalactam $(91 \%)$. (18-19).

Los hallazgos bacteriológicos muestran un importante predominio de $S$. aureus lo cual nos alerta sobre la posibilidad de que estas infecciones sean adquiridas en el medio hospitalario a pesar de ser sensibles a los antibióticos estudiados, no se puede descartar esta posibilidad por no haberse realizado estudio de producción de b lactamasa o sensibilidad a meticilinas. Además, aunque se utilizó el catéter de triple luz modificado para la toma de las muestras endometriales, no se puede descartar la contaminación vaginal como fuente de este microorganismo. La masiva presencia de gérmenes anaerobios aislados en la cavidad endometrial de las pacientes incluidas en este estudio, confirma la necesidad de cobertura para estos microorganismos en la escogencia del esquema antibiótico. Los resultados finales obtenidos demuestran que las dos alternativas cubren adecuadamente este espectro. Se considera importante intensificar la búsqueda de gérmenes causantes de enfermedades de transmisión sexual en las pacientes que acuden a parto al Instituto Materno Infantil.

A pesar del tamaño reducido de la muestra, no se observaron reacciones adversas serias en ninguno de los dos grupos de tratamiento.

\section{Conclusiones}

La endometriosis post-cesárea que se presentó durante el período comprendido entre septiembre de 1993 y agosto de 1994 en el Instituto Materno Infantil de Santa Fe de Bogotá es una infección polimicrobiana con importante participación de gérmenes anaerobios. La asociación clindamicina-gentamicina fue tan eficaz como la asociación pefloxacina-metronidazol en el tratamiento de la endometritis post-cesárea por lo que ésta última asociación se podrá tener como una alternativa en pacientes con fracaso terapéutico en el primer esquema o en pacientes con infecciones severas.

\section{Agradecimientos}

A la Dra. Edith Angel, a los residentes de Obstetricia y Ginecología y al personal de Sépticas del Instituto Materno Infantil.

\section{BIBLIOGRAFIA}

1. Benson CR. Diagnóstico y tratamiento ginecoobstétricos 3ra. edición. Manual Moderno 1982. México.

2. Galba J., Chagas F. Lugar de la operación cesárea y elección del método. Clin. Obstet. Ginecol. Temas actuales. 1982; 3: 727.

3. Guzmán A., Gaitán H. Operación cesárea en el Hospital Materno Infantil en 1984. Trabajo de promoción.

4. Gaitán H., Vargas G. Endometriosis postcesárea tratamiento médicoquirúrgico vs. tratamiento médico en el Instituto Materno Infantil de Bogotá del 1o. de agosto de 1990 al 31 de julio de 1991. Controversias en Ginecología, Obstetricia y Perinatología. Experiencia Institucional UN-IMI 1993. Ediciones Lernier Santa Fe de Bogotá, 1994.

5. Eschembach D., Wager G. Infecciones puerperales. Clin. Obstet. Ginecol. 1981.

6. Stevenson C., Behney C., Miller N. Maternal Death from Puerperal Sepsis Following Cesarean Section Obst. \& Gynec. 1967; 29: 181.

7. Gilstrap L., Cunningham $G$. The bacterial pathogenesis of infection following cesarean section. Obst. \& Gynec. 1979; 53: 545.

8. Monif G. Enfermedades infecciosas en Obstetricia y Ginecología. Salvat Barcelona 1985.

9. Eschenbach D. Acute Postpartum Infections. Emergency Med. Clinics of North America 1985; 3(1): 87.

10. Gibbs R., Jones P., Wilder C. Antibiotic therapy of Endometritis following cesarean section Obst. \& Gyne 1978; 52: 31.

11. Dizerega GS., Yonekura a ML., Roy S et al. A comparison of clindamycin-gentamicin and penicillin-gentamicin in the treatment of post-cesarean section endomyometritis. Am. J. Obstet. Gynecol. 1979; 134: 238.

12. Yonekura ML. Treatment of postcesarean endomyometritis Clin. Obst. Gynec. 1988; 31: 488-500.

13. Sweet R., Gibbs R. Infectius diseases of the Female genital tract. Baltimore Williams Wilkins, 1990 2nd Edition.

14. Katzung EG. Basic and Clinical Pharmacology: Apleton \& Lange 1992.

15. Arredondo J., Orrantia R., Santana H. Tratamiento de la endometritis postcesárea y postparto. Comparación de dos esquemas: pefloxacina vs ampicilina gentamicina. Ginec. Obstet. Mex. 1990; 58: 87.

16. Gaitán H., Villarreal J. Manejo de la infección post-operatoria de la cesárea en el Hospital Materno Infantil del 1o. de marzo de 1985 al 31 de enero de 1986. Manuscrito en preparación.

17. Marens M., Faro S., Hammill. Sulbactam ampicillin versus metronidazole gentamicin in the treatment of postcesarean section endometritis. Diagn. Microbiol. Infect. Dis. 1989; 12: 189s.

18. Gibbs R., Blanco J., Duff P., Castañeda Y., Clair B. A double-blind randomized comparison of moxalactum versus clindamicyngentamicina in the treatment of endomyometritis after cesarean section delivery. Am. J. Obst. \& Gynec. 1983; 146: 769.

19. Gibbs R., Blanco J., Castañeda Y., Clair P. A doble blind randomized comparison of clindamycin-gentamicin versus cefamandole for treatment of post-cesarean section endomyometritis. Am. J. Obst. \& Ginec. 1982; 144: 261. 